\title{
Status of DUNE
}

A. Tonazzo ${ }^{1 *}$, on behalf of the DUNE Collaboration

1 APC, Univ Paris Diderot, CNRS/IN2P3, CEA/Irfu, Obs de Paris, Sorbonne Paris

Cité, France

* tonazzo@in2p3.fr

October 26, 2018

Proceedings for the 15th International Workshop on Tau Lepton Physics, Amsterdam, The Netherlands, 24-28 September 2018

scipost.org/SciPostPhysProc.Tau2018

\begin{abstract}
The Deep Underground Neutrino Experiment (DUNE) is a next-generation underground observatory, to be located in the USA, aiming at precise measurement of long-baseline neutrino oscillations over a $1300 \mathrm{~km}$ baseline, detection of supernova neutrinos and search for nucleon decay and other physics beyond the Standard Model. The far detector, a very large liquid argon time projection chamber, requires a dedicated prototyping effort (ProtoDUNE), currently ongoing at CERN.
\end{abstract}

\section{Contents}

\begin{tabular}{lll}
\hline 1 & Introduction & 1
\end{tabular}

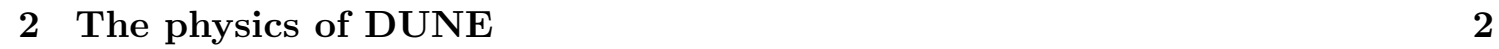

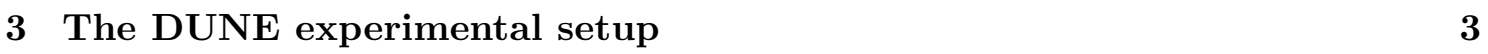

\begin{tabular}{|lll}
4 & The ProtoDUNE detector prototypes at CERN & 4
\end{tabular}

5 Conclusion $\quad 5$

\begin{tabular}{lr}
\hline References & 6
\end{tabular}

\section{Introduction}

The Deep Underground Neutrino Experiment (DUNE) will consist of a far detector located deep underground, at the Sanford Underground Research Facility (SURF) in South Dakota, USA, and a near detector at Fermilab in Illinois. It will study neutrino oscillations over a $1300 \mathrm{~km}$ baseline, using a wide-band neutrino beam produced at the Fermilab accelerator complex, with the main goal of determining the neutrino mass ordering and 

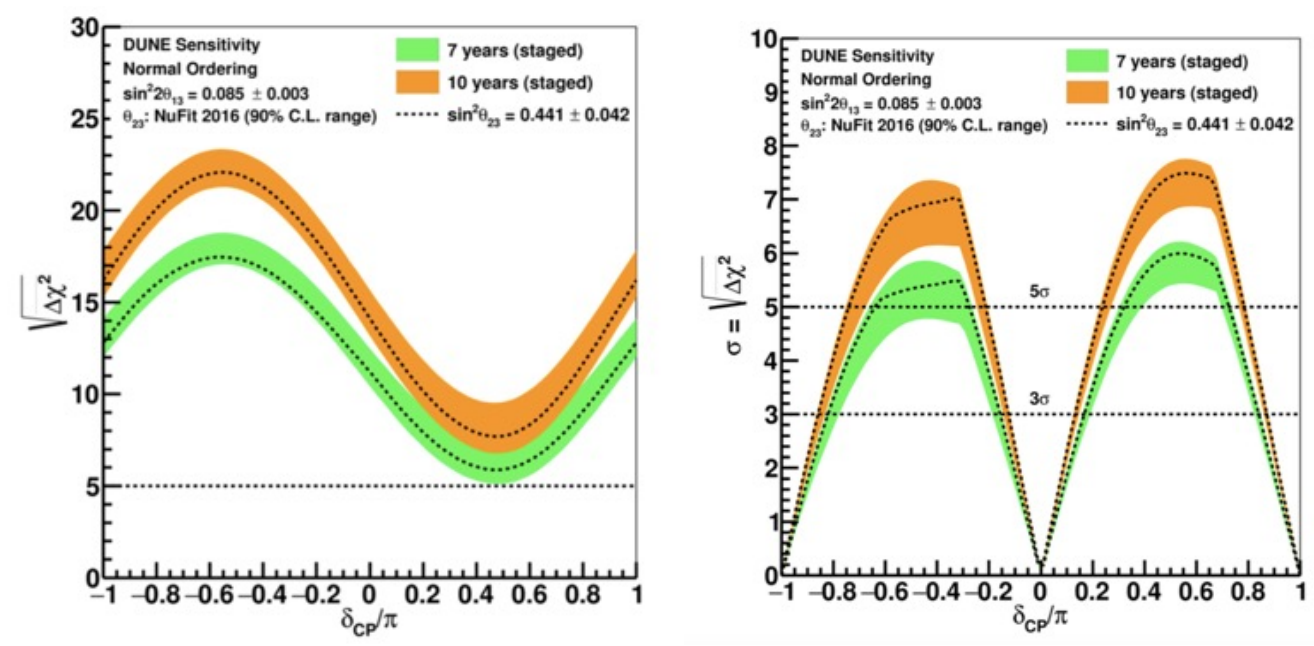

Figure 1: Sensitivity of DUNE to the neutrino mass ordering (left) and the leptonic CP violation phase (right). The staging approached is described in Section 3. The green and orange bands correspond to an exposure of 300 and $556 \mathrm{kt} \times \mathrm{MW} \times \mathrm{y}$ respectively. From Ref. [2].

searching for $\mathrm{CP}$ violation in the leptonic sector, as well as measuring precisely the oscillation parameters, notably the $\theta_{23}$ octant. It will also offer interesting opportunities to search for physics beyond the Standard Model, such as baryon number violation or searches for new particles. In addition, it will detect supernova bursts, solar and atmospheric neutrinos.

The far detector will be a modular liquid argon time projection chamber (LArTPC) with a total fiducial mass of about 40 kton. The construction will follow a modular approach, with the first 10-kt module constructed by 2024. Two technologies are envisaged, Single Phase (SP) and Dual Phase (DP). Both of them require a dedicated prototyping efforts, to test technical solutions, construction procedures and reconstruction/analysis algorithms on a large scale. The ProtoDUNE detectors are being constructed with this purpose at CERN, in the context of the Neutrino Platform. They will be exposed to particle beams, to test the physics performances and provide measurements of the interaction cross-sections on Ar nuclei.

\section{The physics of DUNE}

The DUNE experiment will measure 1,2 muon-neutrino disappearance and electron and tau neutrino appearance, with both neutrino and anti-neutrino beams, providing information on several neutrino oscillation parameters in a single experiment. At the chosen baseline distance of $1300 \mathrm{~km}$, the effects of matter-induced asymmetry and of the CP violation phase $\delta_{C P}$ impact the neutrino spectra differently, thus the two effects can be disentangled. In 10 years of operation, DUNE will reach $5 \sigma$ sensitivity to the neutrino mass ordering for any value of $\delta_{C P}$ and it will be able to discover $\mathrm{CP}$ violation in the leptonic sector at $3 \sigma$ for $50 \%$ of $\delta_{C P}$ values. The sensitivities are shown in Figure 1. Improvements are expected with recent analyses on full simulation, using automated energy reconstruction and deep-learning based event selection.

Another primary physics goal of DUNE is the search for nucleon decay in several important decay modes. The most interesting one in DUNE is $p \rightarrow K^{+} \bar{\nu}$, which is expected 
to have a lifetime of the order of $>10^{33}$ years in SUSY models. Other decay modes, such as $n \rightarrow K^{+} e, p \rightarrow l^{+} K^{0}$ and $p \rightarrow \pi^{0} e^{+}$, are also being studied. Neutron-antineutron oscillations are another possible manifestation of baryon-number non-conservation, on which studies have started.

DUNE will detect and measure of the flux of neutrinos from a core-collapse supernova, should one occur in our galaxy during the lifetime of the experiment. It will have unique sensitivity to the $\nu_{e}$ emitted in the early phases of the core-collapse, via the absorption interaction on ${ }^{40} \mathrm{Ar}: \nu_{e}+{ }^{40} \mathrm{Ar} \rightarrow e^{-}+{ }^{40} K^{*}$.

A variety of opportunities for physics beyond the Standard Model will be provided by the combination of a high-intensity neutrino beam with the near detectors and the massive fare detectors. DUNE will be able to search for new particles, such as low-mass dark matter particles produced in the beam and interacting in the near detector, boosted dark matter produced in the sun or in the galactic center and scattering inelastically in the far detector, heavy neutral leptons originating from the decay of charm and bottom mesons in the beam and detected in the near detectors. DUNE will also explore possible deviations from the PMNS neutrino mixing paradigm, by searching for the effects of nonstandard neutrino interactions, non-unitarity, violations of Lorentz or CPT symmetry or large extra-dimensions in long-baseline oscillations or for active strike neutrino mixing or neutrino trident production in the near detector.

\section{The DUNE experimental setup}

The neutrino beam for DUNE [3] will be an intense wide-band beam produced by $60-120$ $\mathrm{GeV}$ protons from Fermilab's main injector. The spectrum has been optimised for DUNE oscillation physics goals. Operation will start in 2026 with a power of $1.2 \mathrm{MW}$, upgradable to $2.4 \mathrm{MW}$ by 2032 . Both neutrino and anti-neutrino modes will be possible.

The near detector complex, whose role is to constrain systematic uncertainties for oscillations by measuring $\nu$ fluxes to few $\%$ and interaction cross-sections, will be installed at about $600 \mathrm{~m}$ from the target. It will consist of multiple systems: a highly segmented liquid Argon TPC, with the same target material as the far detector, followed downstream by a magnetised multi-purpose tracker with a calorimeter and a muon spectrometer. A movable detector concept is proposed, for off-axis measurements. The conceptual design of the near detector is being finalised.

The DUNE far detector 2, 4, will be located at the Sanford Underground Research Laboratory in South Dakota, USA, at a depth of about $1500 \mathrm{~m}$. The groundbreaking ceremony for the project took place in July 2017 and excavation for the detector hall is currently in progress.

Liquid argon will serve both as target and as detection medium. The detector will consist of 4 modules, using the time projection chamber (TPC) technology, with a fiducial mass of about $10 \mathrm{kt}$ each. A staged construction is envisaged, with the first detector module operational by 2024, $20 \mathrm{kt}$ in 2026, $30 \mathrm{kt}$ in 2027 and the full $40 \mathrm{kt}$ in 2029 .

The interaction of a charged particle in liquid argon produces both scintillation and ionisation. Scintillation light is used mainly for triggering purposes. Ionisation electrons are drifted by an electric field $(\sim 500 \mathrm{~V} / \mathrm{cm})$ to the readout planes, which provide position, timing and energy of the event. LAr-TPCs are characterised by a large and homogeneous active volume, $3 \mathrm{D}$ imaging with $\mathrm{mm}$ resolution in the three coordinates, accurate calorimetry and particle identification from $d E / d x$ measurements and from event topology.

Two designs are envisaged for the far detector modules and shown schematically in Figure 2, In the Single-Phase (SP) option [5,6], electrons drift horizontally over a $3.6 \mathrm{~m}$ 

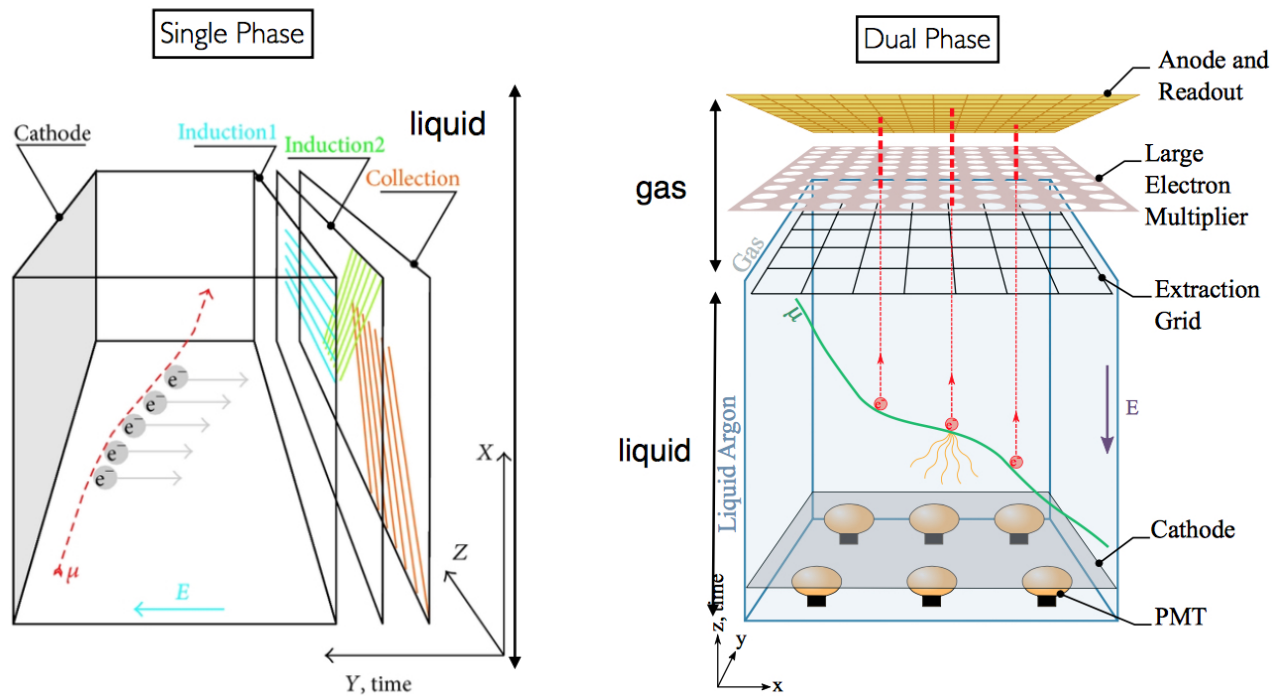

Figure 2: Schematic view of the operating principle of the DUNE Single-Phase (left) and Dual-Phase (right) liquid argon TPCs.

distance and are collected by anode wires immersed in the LAr, with one collection and two tilted $\left( \pm 37.7^{\circ}\right)$ induction views with $5 \mathrm{~mm}$ pitch. The photon detectors are composed of light guides read out by SiPM, embedded in the Anode Readout plane Assemblies (APAs). The Dual-Phase (DP) approach 7 envisages vertical drift over a distance of about $12 \mathrm{~m}$. The ionisation electrons are extracted from Large to a gaseous volume, where the signal is amplified by Large Electron Multipliers (LEM) and the charge is collected on Cathode Readout Planes (CRPs) having two orthogonal views with $3 \mathrm{~mm}$ pitch. Scintillation photons are detected by an array of photomultipliers placed below the cathode.

The realisation of very large scale detectors requires a dedicated prototyping effort, that will be presented in the next section.

\section{The ProtoDUNE detector prototypes at CERN}

The construction of 10-ktonne scale LAr TPCs requires large-scale prototypes, with fiducial mass of the order of 300 tonnes, to test technical solutions, such as production and installation procedure and operation, as well as to validate the performance and the long term behaviour. Two prototypes are being constructed and operated at CERN, Switzerland, in the context of the CERN Neutrino Platform. A dedicated extension of the experimental North Area has been constructed, where the two cryostats have been installed and equipped. The prototypes will be exposed to cosmic rays and to particle beams $(p, \pi, K, e$ with $0.5-20 \mathrm{GeV}$ momentum) from the SPS complex, to test their performance and to carry on physics studies, such as validation of reconstruction and particle identification algorithms and measurement of hadron interaction cross-sections on argon nuclei.

ProtoDUNE Single-Phase has a fiducial volume of $6.9 \times 7.2 \times 6 \mathrm{~m}^{3}$, separated vertically in two drift regions by the Cathode Assembly Plane. Detector elements were completed, tested and inserted by the end of April, 2018. Filling with LAr was completed in about one month, on September $13^{\text {th }}, 2018$. Purity monitors and temperature sensors were operational during filling. The detector successfully recorded the first tracks from cosmic rays, such as the one shown in Fig. 3, as announced in a CERN press release 8. 


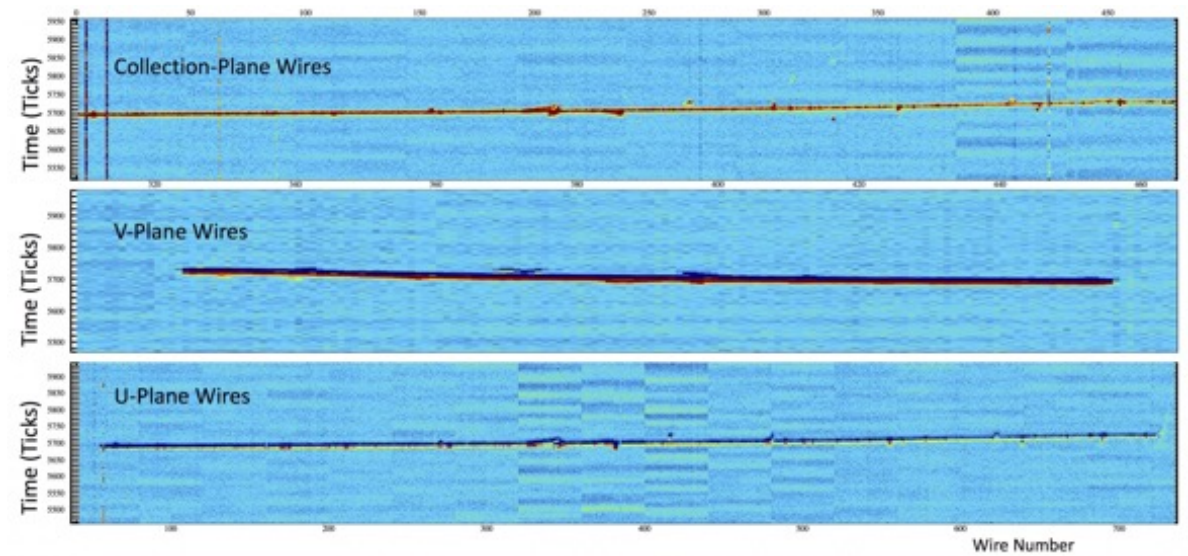

Figure 3: One of the first cosmic muon particle tracks, with about $3.8 \mathrm{~m}$ length, observed by the ProtoDUNE detector at CERN. The signal is recorded on three wire planes. From Ref. [8].

Data taking in beam trigger mode is ongoing at the time of writing.

ProtoDUNE Dual-Phase consists of a single active volume of $6 \times 6 \times 6 \mathrm{~m}^{3}$. The field cage for the drift field was completed and tested in April 2018. At the time of writing, construction and test of the Cathode Readout Planes is ongoing. Commissioning with 2 CRPs fully instrumented is foreseen early 2019.

A smaller demonstrator 9 for the ProtoDUNE Dual-Phase technology, with a fiducial volume of $3 \times 1 \times 1 \mathrm{~m}^{3}$ (4 tonne fiducial mass), was constructed and operated at CERN in 2017. $5 \times 10^{5}$ interactions of cosmic events were recorded. First results have been obtained on charge amplification, light detection, and purity of liquid argon, a crucial parameter to ensure the capability of detecting the ionisation charge after a 12-m drift. An electron lifetime of the order of $4 \mathrm{~ms}$ has been measured, which is adequate for the DUNE requirements.

\section{Conclusion}

DUNE will measure neutrino and anti-neutrino oscillations over $1300 \mathrm{~km}$ baseline. Large underground detectors will provide further interesting opportunities: search for nucleon decay and other physics beyond the standard model, detection of a supernova burst.

The far detector will be a large, modular $(4 \times 10$ ktonne fiducial mass $)$ liquid argon TPC, with Single- and Dual-Phase technology. Large-scale detector prototypes, ProtoDUNE, are being tested at CERN.

The Technical Design Report for DUNE will appear in spring 2019. The first module of the far detector will be ready ready in 2024 and physics physics with the beam will start in 2026.

\section{Acknowledgements}

We are indebted to the organisers of the TAU2018 Workshop for giving us the opportunity to present the status of DUNE. 


\section{References}

[1] DUNE Collaboration, Long-Baseline Neutrino Facility (LBNF) and Deep Underground Neutrino Experiment (DUNE) : Conceptual Design Report, Volume 2: The Physics Program for DUNE at LBNF , arXiv:1512.06148 [physics.ins-det]

[2] DUNE Collaboration, The DUNE Far Detector Interim Design Report, Volume 1: Physics, Technology and Strategies, arXiv:1807.10334 [physics.ins-det]

[3] DUNE Collaboration, Long-Baseline Neutrino Facility (LBNF) and Deep Underground Neutrino Experiment (DUNE) : Conceptual Design Report, Volume 1: The LBNF and DUNE Projects, arXiv:1601.05471 [physics.ins-det]

[4] DUNE Collaboration, Long-Baseline Neutrino Facility (LBNF) and Deep Underground Neutrino Experiment (DUNE) : Conceptual Design Report, Volume 4: The DUNE Detectors at LBNF, arXiv:1601.02984 [physics.ins-det]

[5] DUNE Collaboration, The Single-Phase ProtoDUNE Technical Design Report , arXiv:1706.07081 [physics.ins-det]

[6] DUNE Collaboration, The DUNE Far Detector Interim Design Report, Volume 2: Single-Phase Module, arXiv:1807.10327 [physics.ins-det]

[7] DUNE Collaboration, The DUNE Far Detector Interim Design Report, Volume 3: Dual-Phase Module, arXiv:1807.10340 [physics.ins-det]

[8] CERN press release, 18/09/2018, https://home.cern/about/updates/2018/09/firstparticle-tracks-international-neutrino-experiment

[9] B. Aimard et al., arXiv:1806.03317 [physics.ins-det], to be published in JINST 